\title{
Diagnósticos Enfermeros prevalentes en pacientes mayores con úlceras por presión: estudio transversal
}

\author{
Prevalent nursing diagnoses in elderly with \\ pressure ulcers: cross-sectional study
}

\section{Diagnósticos Enfermeiros prevalentes em pacientes idosos com úlceras por pressão: estudo transversal}

\author{
E. Álvarez-Bolaños ${ }^{\mathrm{a} 1}$, B.E. Cegueda-Benítez ${ }^{\mathrm{b} 2}$, M.A. Cuevas-Budhart ${ }^{\mathrm{c} 3,5 *}$,

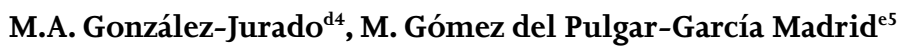 \\ ORCID: \\ ${ }^{\mathrm{a}} 0000-0002-9966-1145 \quad{ }^{\mathrm{b}} 0000-0002-2008-8113 \quad{ }^{\mathrm{c}} 0000-0002-3379-0147$ \\ ${ }^{\mathrm{d}} 0000-0002-5282-4517 \quad{ }^{\mathrm{e}} 0000-0002-6323-1020$ \\ ${ }^{1}$ Coordinación Delegacional de Enfermería en Atención Médica, IMSS, Guerrero, México \\ ${ }^{2}$ UMAE “Dr. Victorio de la Fuente Narváez”, Ciudad de México, México \\ ${ }^{3}$ Departamento de Enfermería, Hospital General Regional No 1 "Vicente Guerrero”, IMSS, Acapulco Guerrero, \\ México \\ ${ }^{4}$ Consejo General de Enfermería de España, Madrid, España \\ ${ }^{5}$ Instituto Español de Investigación Enfermera, Madrid, España
}

Recibido: 16 febrero 2019

Aceptado: 30 mayo 2019

\section{Resumen}

Objetivo: Determinar la prevalencia de los diagnósticos enfermeros en pacientes adultos mayores hospitalizados con úlceras por presión e identificar los diagnósticos enfermeros reales, potenciales, de salud y síndrome por patrón funcional de salud. Material y métodos: Estudio Transversal, descriptivo conformado por una muestra de pacientes adultos mayores hospitalizados en diferentes servicios de un Hospital de Acapulco, Guerrero, México. Para su evaluación, se diseñó un instrumento de acuerdo con los patrones funcionales de Marjory Gordon. Se utilizó la plataforma de mejores prácticas de enfermería E-cuidados® para el análisis.

Resultados: El presente estudio identificó 36 diagnósticos relevantes, de los cuales 23 (63.9 \%) fueron diagnósticos centrados en el problema, 10 (27.8 \%) diagnósticos de riesgo y 3 (8.3 \%) de promoción 
a la salud. Los patrones funcionales más afectados son; Actividad-Ejercicio, Nutricional-Metabólico y Valores-Creencias.

Conclusiones: El presente estudio ayuda a enfatizar la importancia de adoptar estrategias preventivas y de atención integral, así como la mejora de la planificación de la atención para evitar el deterioro de las condiciones en los pacientes adultos mayores.

Palabras clave: Adulto mayor; diagnósticos de enfermería; úlceras por presión; México.

\section{Abstract}

Objective: To determine the prevalence of diverse nursing diagnostics in hospitalized older patients with pressure ulcers, identifying the real, potential, and health-related ones, as well as syndrome by health functional pattern.

Methods and material: This is a transversal and descriptive study with a sample of older patients in different services in a hospital of Acapulco, Guerrero, Mexico. An assessment instrument was designed following the Functional Patterns of Marjory Gordon. The E-cuidados ${ }^{\circledR}$ best nursing practices platform was used for the analysis.

Results: 36 relevant diagnostics were identified: 23 (63.9\%) were problem-centered; $10(27.8 \%)$ were risk related; and $3(8.3 \%)$ were health promoting. The most affected functional patterns were: Activity-Exercise; Nutrition-Metabolism; and Values-Beliefs.

Conclusions: This study supports the need to highlight the importance of adopting preventive and integral attention strategies, as well as improving the attention planning process in order to avoid the deterioration of health conditions of older patients.

Keywords: Aged; nursing diagnosis; pressure ulcers; Mexico.

\section{Resumo}

Objetivo: Determinar a prevalência dos diagnósticos enfermeiros em pacientes idosos hospitalizados com úlceras por pressão e identificar os diagnósticos enfermeiros reais, potenciais, de saúde e síndrome por padrão funcional de saúde. Material e métodos: Estudo Transversal, descritivo conformado por uma amostra de pacientes idosos hospitalizados em diferentes serviços de um Hospital de Acapulco, Guerrero, México. Para sua avaliação, desenhou-se um instrumento conforme com os padrões funcionais de Marjory Gordon. Utilizou-se a plataforma de melhores práticas de enfermagem E-cuidados ${ }^{\circledR}$ para a análise.

Resultados: O presente estudo identificou 36 diagnósticos relevantes, dos quais 23 (63.9\%) foram diagnósticos centrados no problema, 10 (27.8\%) diagnósticos de risco e $3(8.3 \%)$ de promoção à saúde. Os padrões funcionais mais afetados são; Atividade-Exercício, Nutricional-Metabólico e Valores-Crenças. Conclusões: O presente estudo ajuda a enfatizar a importância de adotar estratégias preventivas e de atenção integral, assim como a melhora da planificação da atenção para evitar a deterioração das condições nos pacientes idosos.

Palavras chave: Idoso; diagnósticos de enfermagem; úlceras por pressão; México.

\section{Introducción}

Las úlceras por presión (UPP) suponen un problema de salud a nivel Nacional, dado que, prolongan las estancias hospitalarias ${ }^{1}$ e incrementan los costos de los procesos del sistema sanitario, constituyen un indicador negativo de la institución, ya que el $95 \%$ de ellas pueden ser evitables, es decir, este porcentaje puede ser atribuible al déficit de la calidad asistencial. Los mecanismos que causan las UPP son innumerables y precisan ser atenuados o prevenidos, completamente, para que la integridad cutánea sea mantenida ${ }^{2,3}$.

Los pacientes con mayor riesgo de presentar UPP son aquellos confinados en cama por períodos prolongados, con disfunción motora y sensitiva, así como los pacientes bajo efectos de sedación, presencia 
de edema y atrofia muscular ${ }^{4}$; los adultos mayores (AM) $)^{1 *}$ pueden presentar algunas de estas condiciones, por esta razón son considerados una población vulnerable, ya que los cambios naturales del envejecimiento y los problemas de salud se traducen en un deterioro de las capacidades funcionales, mentales y sociales ${ }^{7}$. Por otra parte, en México, el envejecimiento poblacional se caracteriza por la coexistencia de enfermedades no transmisibles (ENT), enfermedades transmisibles y desnutrición ${ }^{8}$, esto hace de los ancianos, susceptibles a hospitalizaciones y en consecuencia a riesgo de UPP; además, este riesgo se vincula con el estado de la piel y la nutrición del paciente AM; otros factores asociados a las UPP son la edad, fricción, humedad, estado de conciencia y procesos patológicos terminales 9 .

Por lo tanto, resulta relevante evaluar esta condición tan frecuente como son las UPP, en una población con alto riesgo de presentarlas, a fin de mejorar la calidad y seguridad de la atención a este grupo. La evaluación más idónea puede ser con el uso de la herramienta metodológica propia de la disciplina, esto es el Proceso de Atención de Enfermería.

De acuerdo a la Asociación Americana de Enfermería (ANA), el proceso enfermero es considerado como un estándar para la práctica enfermera, su importancia ha exigido cambios sustanciales en sus etapas, que favorecen al desarrollo de la profesión como disciplina científica, lo cual, ha incrementado la calidad en la atención ${ }^{10-12}$.

El proceso enfermero, consta de cinco etapas, las cuales, se encuentran estrechamente relacionadas, estas son; valoración, diagnóstico, planeación, ejecución y evaluación. Para este estudio nos referiremos sólo a las dos primeras etapas.

La identificación de los diagnósticos de enfermería, mediante una correcta valoración, permite describir los principales problemas y necesidades de los pacientes para brindar una atención segura y de calidad, así mismo reducir los días de estancia hospitalaria, costos y complicaciones ${ }^{10-14}$. El Proceso de Atención de Enfermería, favorece a la identidad de la profesión, la claridad de los roles, la investigación y estandarización del cuidado enfermero. Este último aspecto es recomendado por la NANDA Internacional, para la organización de los datos que orientan los cuidados, que contribuyen a mantener y recuperar la salud.

Por otra parte, se consideró que los 11 patrones funcionales de salud descritos por Marjory Gordon, son configuraciones del comportamiento, construidos desde la descripción del paciente y las observaciones del profesional de Enfermería. La utilización de estos patrones funcionales, permite una valoración sistemática con la que se obtienen datos relevantes del paciente, se detectan posibles anomalías o riesgos de alteración, de este modo se determinan los principales diagnósticos de enfermería.

Estos diagnósticos, se clasifican en; centrados en el problema (reales), de riesgo, síndrome y de promoción de la salud ${ }^{15,16}$

En este contexto, se han desarrollado diversos estudios, los cuales exploran los diagnósticos enfermeros prevalentes o frecuentes de ciertas patologías específicas, como; pénfigo vulgar ${ }^{17}, \mathrm{VIH} / \mathrm{SIDA}^{18}$, inclusive de manera general en los pacientes que padecen enfermedades crónico-degenerativas ${ }^{19}$, por otro lado, existen estudios en servicios hospitalarios específicos: urgencias ${ }^{20}$, traumatología y cirugía ${ }^{21}$, hematología y oncología ${ }^{22}$, psiquiatría hospitalariaa ${ }^{23}$, e incluso en dolor agudo en niños hospitalizados ${ }^{24}$. También, el grupo de pacientes que se encuentran bajo un tratamiento sustitutivo como; hemodiálisis ${ }^{25}$ o trasplante renal ${ }^{26}$, además en ciertos padecimientos, angina refractaria con diagnóstico de intolerancia a la actividad ${ }^{27}$, es uno de ellos.

Como se puede observar, son diversos los ámbitos en los que se describe la clasificación NANDA ${ }^{15}$, ya que, es lo que nos identifica como profesión, además este tipo de estudios estimula la investigación al igual que enriquece el desarrollo de la profesión.

Es por ello, que la identificación de la prevalencia de diagnósticos en enfermería en pacientes adultos mayores hospitalizados que presentan UPP, posibilitaría mejorar la calidad de la atención y seguridad del

1 * Las Naciones Unidas considera anciano a toda persona mayor de 65 años para los países desarrollados y de 60 a más para los países en desarrollo, tal es el caso de México ${ }^{5,6}$

Diagnósticos Enfermeros en pacientes mayores con úlceras por presión • E. Álvarez-Bolaños et al. 
paciente, además de servir como guía para la implementación de planes de cuidado complementarios, en este contexto, ante los problemas de salud reales o potenciales, desarrollar la práctica de los cuidados de enfermería mediante la reflexión crítica en la atención individualizada de los pacientes.

Desde esta perspectiva permite sistematizar y estandarizar el cuidado con criterios unificados que hacen más objetivo el actuar del profesional de Enfermería. Esto se puede traducir en una prevención eficaz de las ulceras por presión, lo que beneficia no sólo al usuario, sino, a la institución prestadora del servicio.

Por lo antes mencionado, el objetivo de este estudio fue determinar la prevalencia de los diagnósticos enfermeros en adultos mayores hospitalizados con úlceras por presión e identificar los diagnósticos enfermeros reales, potenciales, síndrome y, los de salud basados en los patrones funcionales.

\section{Metodología}

Se realizó un estudio descriptivo y transversal entre noviembre de 2016 y febrero de 2017. Los criterios de selección fueron: pacientes adultos mayores de 60 años, pacientes con seis o más días de hospitalización, con diversa patología, hospitalizados en los servicios de Medicina Interna, Traumatología y Ortopedia, Nefrología, Cirugía, Urgencias y Unidad de Cuidados Intensivos en un Hospital General en Acapulco, Guerrero, México, y que presentaran UPP. Por lo cual, el muestreo fue no probabilístico, por serie de casos consecutivos.

Para la recolección de la información, se diseñó un instrumento basado en el proceso de atención de enfermería así como en los 11 patrones funcionales de salud de Marjory Gordon ${ }^{28}$, lo que demuestra una validez inductiva, es decir, la credibilidad (Trustworthiness) y la confiabilidad de los datos y su interpretación, los cuales se distinguen por validez descriptiva, interpretativa y teórica ${ }^{29,30}$. La validez lógica se realizó mediante el método Delphi con expertos en el área, quienes ocupan puestos gerenciales en instituciones públicas del sector de salud con amplia experiencia en validación de instrumentos y registros clínicos de Enfermería, con lo cual, se obtuvo un instrumento de 131 ítems de respuesta dicotómica, el cual, contaba con las siguientes dimensiones; Datos socio-demográficos (8 ítems), Patrón Funcional 1, Percepción-Manejo de la Salud (15 ítems), Patrón Funcional 2, Nutricional-Metabólico (18 ítems), Patrón Funcional 3, Eliminación (18 ítems), Patrón Funcional 4, Actividad-Ejercicio (12 ítems), Patrón Funcional 5, Sueño-Reposo (8 ítems), Patrón Funcional 6, Cognitivo-Perceptual (19 ítems), Patrón Funcional 7, Autopercepción-Autoconcepto (10 ítems), Patrón Funcional 8, Rol-Relaciones (11 ítems), Patrón Funcional 9, Sexualidad-Reproducción (8 ítems), Patrón Funcional 10, Afrontamiento-Tolerancia al estrés (7 ítems), Patrón Funcional 11, Valores-Creencias ( 5 ítems), posteriormente, se realizó la validez de contenido, se obtuvo una consistencia interna de 0.96; cabe señalar, que el instrumento fue diseñado y validado para fines exclusivos de este estudio. Para agrupar las características de las UPP, se utilizó la Clasificación y estadios de las Úlceras por Presión conforme a los lineamientos establecidos por el Grupo Nacional para el Estudio y Asesoramiento en Úlceras por presión y Heridas Crónicas².

Confirmada la validez del instrumento, se procedió a capacitar a dos pasantes del servicio social de la licenciatura de Enfermería para realizar la valoración por patrones funcionales de salud de Marjory Gordon, como parte del Proceso de Atención de Enfermería y el uso correcto del instrumento de recolección de datos para evitar sesgos.

Una vez identificados los pacientes que cumplían con los criterios de selección, fue evaluado el riesgo de UPP y se aplicó el instrumento de valoración de los 11 patrones funcionales de salud de Marjory Gordon, a efecto de identificar los diagnósticos de enfermería más prevalentes. 


\section{Recolección y análisis de datos}

Los datos resultantes, fueron analizados exhaustivamente por los investigadores principales con ayuda de la plataforma E-Cuidados ${ }^{\circledR 1}$ de buenas prácticas enfermeras, lo que permitió la identificación de diagnósticos de enfermería según los criterios establecidos por NANDA Internacional. La plataforma E-Cuidados ${ }^{\circledR 3}$ es una herramienta informática diseñada para la unificación del lenguaje enfermero, creada por el Consejo General de Enfermería de España.

Para el procesamiento de la información se elaboró una base de datos en (SSPS) v.22®; en las variables cuantitativas se utilizaron medidas de tendencia central y dispersión; en cualitativas, frecuencias simples y porcentajes. Para la discusión y análisis de los datos, se elaboraron tablas y gráficos.

\section{Resultados}

Se evaluaron 62 pacientes de los diferentes servicios descritos en los criterios de selección. El mayor porcentaje se reflejó en el rango de edad de 60 a 74 años (43.6 \%), mientras que el menor porcentaje fue en adultos mayores de 85 años $(16.1 \%)$. El género femenino fue el que predominó del total de pacientes evaluados (55\%), más del $50 \%$ cuenta con un nivel básico de estudios, primaria. En relación al estado civil, la mitad son casados, la mayoría profesan religión católica (95\%). (Tabla 1).

Tabla 1. Datos sociodemográficos y clínicos

\begin{tabular}{lc}
\hline Edad (Años) & $\mathrm{f}(\%)$ \\
60 a 74 años & $27(43.6)$ \\
75 a 84 años & $25(40.3)$ \\
$>85$ años & $10(16.1)$ \\
\hline Sexo & \\
Femenino & $34(55.0)$ \\
Masculino & $28(45.0)$ \\
\hline Escolaridad & \\
Preescolar & $1(1.6)$ \\
Primaria & $37(59.7)$ \\
Secundaria & $9(14.5)$ \\
Bachillerato & $10(16.1)$ \\
Universidad & $4(6.5)$ \\
Sin Estudios & $1(1.6)$ \\
\hline Religión & \\
Católica & $59(95.2)$ \\
Cristiana & $2(3.2)$ \\
Otros & $1(1.6)$ \\
\hline Estado Civil & \\
Soltero & $5(8.1)$ \\
Casado & $32(51.6)$ \\
Divorciado & $6(9.7)$ \\
Unión Libre & $12(19.4)$ \\
Viudo & $7(11.3)$ \\
\hline
\end{tabular}

En cuanto a la etiología de la enfermedad, la mayor parte de los pacientes cursaba con alguna enfermedad no transmisible o crónico degenerativa con comorbilidades agregadas (14.5\%), enfermedades cerebrovasculares $(14.5 \%)$, insuficiencia orgánica múltiple $(14.5 \%)$, sepsis $(12.9 \%)$ y fractura de cadera $(12.9 \%)$. (Figura 1$)$. 
- *uúltiples diagnósticos

- Enfermedades cerebrovasculares

- Sepsis

- Fractura de cadera

- Fractura de miembros pélvicos

- Encefalopatías

- Enfermedades card iovasculares

- Descontrol metabólico. Hipertensión

- Complicaciones de la Enfermedad Renal Crón $6 \%$

- DM2 descontrolada

- Traumatismo craneoencefálico

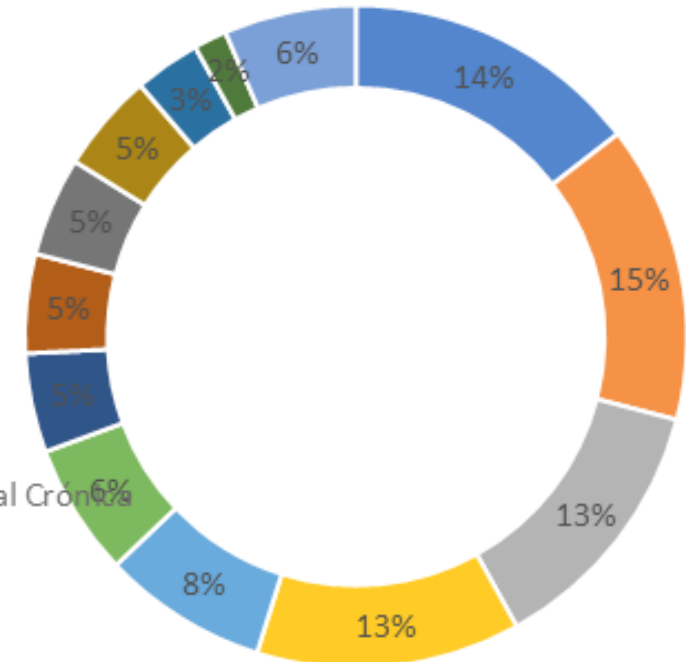

- Neu ropatía diabética

- **otros

${ }^{*} E R C, D M 2$, Has, STDA, Enfermedad Diverticular, Carcinoma, Insuficiencia Cardiaca, Cáncer de Próstata, Derrame Cerebral, Derrame Pleural, Falla Orgánica Múltiple, Acidosis Metabólica, Insuficiencia Respiratoria, Choque Hipovolémico, Tumor Vesical

**Hipercalcemia Severa, Ca Hepático, Hepatitis, Síndrome de Fournier, Miopatía Compresiva

Figura 1. Etiología de la hospitalización.

Los principales resultados clínicos y paraclínicos alterados fueron IMC $\left(\mathrm{kg} / \mathrm{m}^{2}\right)$ fue de $25.6 \pm 5.4$, Hemoglobina g/dl de10.6 \pm 2.2. (Tabla 2).

Tabla 2. Datos clínicos, paraclínicos $(n=62)$

\begin{tabular}{lc}
\hline Constantes vitales & $\mathrm{X} \pm \mathrm{SD}$ \\
Temperatura & $36.6 \pm 0.5$ \\
Frecuencia cardiaca & $80.8 \pm 16.0$ \\
Frecuencia respiratoria & $20.4 \pm 7.5$ \\
Sistólica $(\mathrm{mm} / \mathrm{Hg})$ & $124.5 \pm 30.5$ \\
Diastólica $(\mathrm{mm} / \mathrm{Hg})$ & $72.2 \pm 11.3$ \\
\hline Datos clínicos y paraclínicos & \\
Talla $(\mathrm{cm})$ & $1.61 \pm 0.1$ \\
Peso actual $(\mathrm{kg})$ & $66.4 \pm 13.6$ \\
IMC $\left(\mathrm{Kg} / \mathrm{m}^{2}\right)$ & $25.6 \pm 5.4$ \\
Hemoglobina $(\mathrm{mg} / \mathrm{dl})$ & $10.6 \pm 2.2$ \\
Albumina $(\mathrm{mg} / \mathrm{dl})$ & $3.6 \pm 4.5$ \\
Glucosa $(\mathrm{mg} / \mathrm{dl})$ & $129+77.6$ \\
\hline
\end{tabular}

En relación con la ubicación de las úlceras, el área más afectada fue la región sacra (67.7 \%), seguida de glúteos (6.5 \%) y codos (4.8\%). Según la escala de Braden, el $61.3 \%$ de las lesiones estaban en la etapa I. (Tabla 3). 
Tabla 3. UPP: Región de lesión, estadios

\begin{tabular}{lc}
\hline Región de lesión & $\mathrm{f}(\%)$ \\
Sacra & $42(67.7)$ \\
Glúteo & $4(6.5)$ \\
Codo & $3(4.8)$ \\
Espalda & $3(4.8)$ \\
Talón & $3(4.8)$ \\
Sacra y glúteo & $2(3.2)$ \\
Brazo & $1(1.6)$ \\
Codo izq. & $1(1.6)$ \\
Glúteo derecho y espalda & $1(1.6)$ \\
Hombro & $1(1.6)$ \\
Tobillos & $1(1.6)$ \\
\hline Estadios & \\
Estadio I & $38(61.3)$ \\
Estadio II & $23(37.1)$ \\
Estadio III & $1(1.6)$ \\
\hline
\end{tabular}

Se identificaron 36 diagnósticos de enfermería, 23 se centraron en el problema (63.9\%), 10 diagnósticos de riesgo (27.8 \%) y tres diagnósticos de promoción de la salud (8.3 \%). El riesgo de úlcera por presión y el riesgo de caídas se identificaron en el total de los pacientes estudiados (Tabla 4).

Tabla 4. Diagnósticos de Enfermería

\begin{tabular}{lc}
\hline Diagnósticos & $\%$ \\
\hline Riesgo de úlcera por presión & 100 \\
\hline Riesgo de caídas & 100 \\
\hline Déficit de volumen de líquidos & 90 \\
\hline Deterioro de la religiosidad & 87 \\
\hline Disposición para mejorar el auto concepto & 82 \\
\hline Deterioro de la integridad cutánea & 79 \\
\hline Riesgo de síndrome de desuso & 71 \\
\hline Deterioro de la movilidad física & 66 \\
\hline Deterioro de la integridad tisular & 63 \\
\hline Riesgo de nivel de glicemia inestable & 63 \\
\hline Dolor agudo & 56 \\
\hline Deterioro de la comunicación verbal & 52 \\
\hline Deterioro de la memoria & 45 \\
\hline Deterioro de la respiración espontánea & 42 \\
\hline Sobrepeso & 40 \\
\hline Disposición para mejorar la nutrición & 40 \\
\hline Deterioro de la movilidad en la cama & 39 \\
\hline Exceso de volumen de líquido & 37 \\
\hline
\end{tabular}

\begin{tabular}{lc}
\hline Diagnósticos & $\%$ \\
\hline Insomnio & 35 \\
\hline Ansiedad & 35 \\
\hline Riesgo de infección & 32 \\
\hline Disposición para mejorar la religiosidad & 29 \\
\hline Riesgo de disfunción neurovascular periférica & 27 \\
\hline Sufrimiento espiritual & 26 \\
\hline Baja autoestima situacional & 24 \\
\hline Deterioro de la deglución & 24 \\
\hline Procesos familiares disfuncionales & 21 \\
\hline Riesgo de perfusión renal ineficaz & 21 \\
\hline Deterioro de la interacción social & 14 \\
\hline Estreñimiento & 14 \\
\hline Incontinencia urinaria refleja & 14 \\
\hline Riesgo de perfusión tisular periférica ineficaz & 10 \\
\hline Riesgo de deterioro de la función cardiovascular & 10 \\
\hline Deterioro de la eliminación urinaria & 8 \\
\hline Perfusión tisular periférica ineficaz & 3 \\
\hline Riesgo de disminución de la perfusión tisular cardíaca & 3 \\
\hline
\end{tabular}

Los patrones funcionales de mayor afección fue el patrón 4, Actividad-Ejercicio (28.1\%), seguido de Nutricional-Metabólico correspondiente al patrón número 2 (26.9 \%) y el patrón número 11, Valores-Creencias (9.5\%). (Figura 2). 


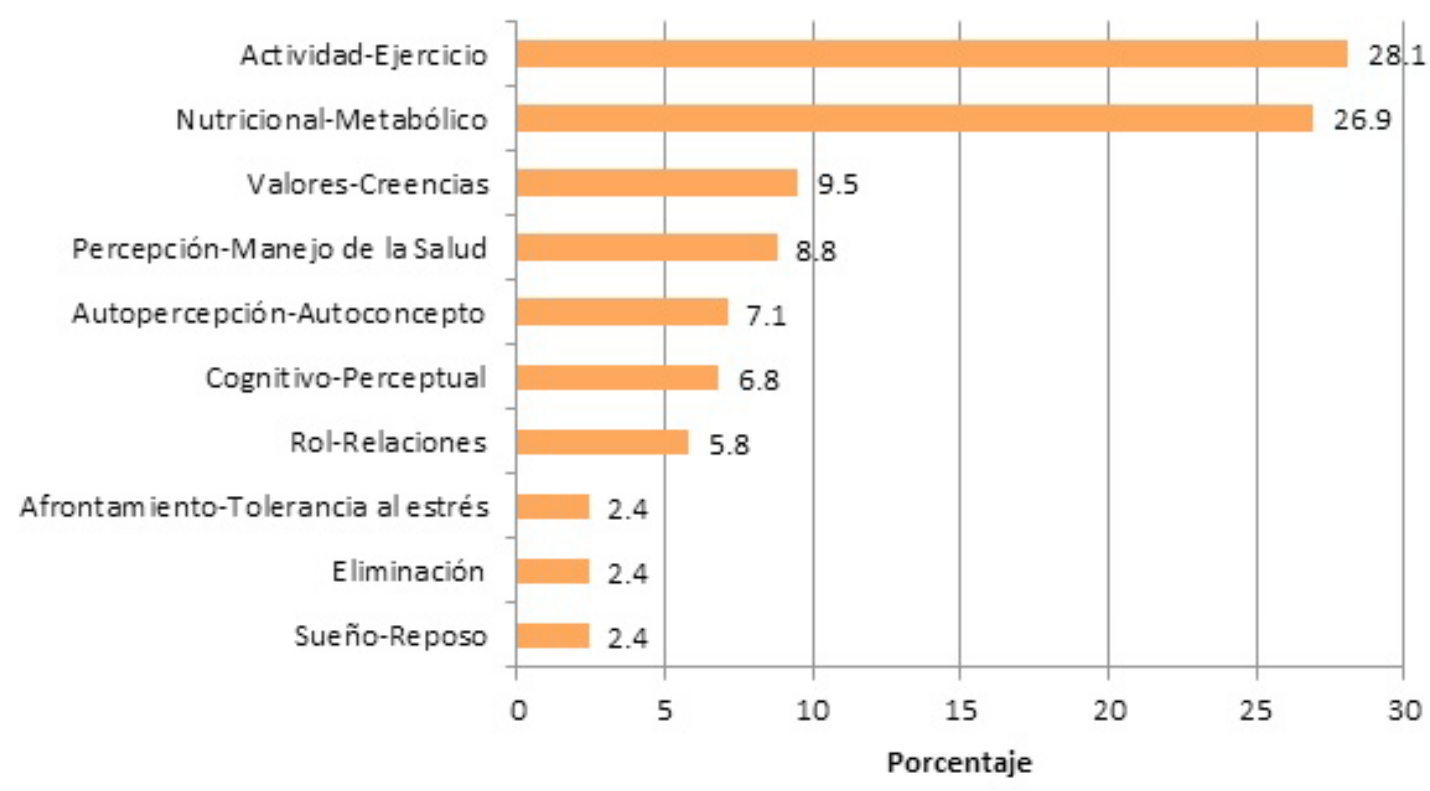

Figura 2. Porcentaje de patrón funcional alterado ( $n=931)$.

\section{Discusión}

Las úlceras por presión (UPP) constituyen un importante problema de salud, consumo de recursos e incluso de responsabilidad legal, por ello, la Organización Mundial de la Salud considera las UPP como un indicador de la calidad asistencial, es decir, de los cuidados de enfermería proporcionados, tanto a pacientes que las presentan, como a los que están en riesgo.

En el Hospital General Regional, se ha presentado una carencia en el cumplimiento de dichos indicadores, lo que demuestra un sistema crítico del cuidado en prevención de ulceras por presión a pacientes hospitalizados y en el porcentaje de pacientes postrados que presentan lesión por presión; los registros clínicos, esquema terapéutico e intervenciones de enfermería carentes de Diagnósticos de Enfermería, evidencian cuidados no estandarizados que provocan UPP.

Dado que la atención del paciente con úlceras por presión debe ser integral (física, emocional, social y espiritual), es importante conocer los diagnósticos de Enfermería con mayor prevalencia en los pacientes hospitalizados, ya que esto contribuye a la mejora de los niveles de evidencia en la aplicación del cuidado enfermero, facilita la planificación de los cuidados más adecuados, anticipa la aparición de necesidades en relación a los diagnósticos identificados de forma sistematizada y permite al profesional enfermero fortalecer su procesos. Estas acciones, pueden ser traducidas a una prevención efectiva de UPP para el paciente y un impacto eficiente para la institución prestadora de servicio en el Sistema Nacional de Salud.

De acuerdo a los resultados de este estudio, encontramos que, los pacientes adultos mayores, cuentan con problemas nutricionales, envejecimiento poblacional sin aumento de la calidad de vida, esto se ve reflejado en los patrones alterados, principalmente, con lo cual, la tendencia de la atención de enfermería debe de ir no sólo enfocada al esquema terapéutico farmacológico, prevención y tratamiento de UPP, sino también a fomentar los hábitos de vida saludables en la dieta, gestionar un vínculo de programas de ejercicios en conjunto con los familiares, según la capacidad del paciente y orientar sobre una la nutrición complementaria.

Más del $50 \%$ de los pacientes tienen el mismo tipo de diagnóstico de enfermería, ya que cursaron con enfermedad crónico degenerativa con comorbilidades agregadas durante su hospitalización, entre los diagnósticos prevalentes de enfermería obtenidos, llama la atención la desnutrición y obesidad, aspectos importantes que condicionan la prevención y tratamiento de las UPP, aunque también es el 
común denominador en la población guerrerense sana y enferma. Según Araújo TM. et al. ${ }^{32}$, hay una mayor frecuencia en diagnóstico centrados en el problema, como: baño/higiene, déficit de autocuidado, movilidad deteriorada y deterioro de la piel. Sin embargo, en el presente estudio, se observa una mayor prevalencia en los diagnósticos de riesgo como úlcera por presión y riesgo de caídas.

Los diagnósticos referidos por Villarejo-Aguilar L. ${ }^{19}$, son similares al presente estudio, ya que coinciden con los cinco primeros de los 14 diagnósticos prevalentes encontrados, su estudio tuvo como objetivo identificar los diagnósticos enfermeros más frecuentes registrados en los Informes de Alta de pacientes con enfermedades crónicas, en gran mayoría adultos mayores, estos son: deterioro de la movilidad física (50.3\%), ansiedad (50.3\%), riesgo de caídas (43\%), riesgo de deterioro de la integridad cutánea $(36.4 \%)$, dolor agudo (18.5\%), intolerancia a la actividad, riesgo de estreñimiento, conocimientos deficientes, cansancio en el desempeño del rol del cuidador, desequilibrio nutricional, manejo inefectivo del régimen terapéutico, riesgo de cansancio en el desempeño del rol de cuidador, afrontamiento familiar comprometido, y déficit de autocuidados: alimentación.

Se observaron como principales diagnósticos de enfermería reales: el déficit de volumen de líquidos y el deterioro de la religiosidad. El primero en relación al diagnóstico médico que predominó, la enfermedad renal crónica derivado del fracaso de los mecanismos reguladores que ocasiona disminución del líquido intravascular, intersticial y/o intracelular, que provoca disminución de la presión arterial, la presión y volumen del pulso, deterioro de la integridad cutánea, sequedad de las membranas mucosas y de la piel.

El deterioro de la religiosidad se ve afectada en la mayoría de la muestra, ya que no pueden asistir a servicios religiosos mientras están hospitalizados. La falta de asistencia a estos servicios puede afectar el comportamiento individual, cambiar la cognición y la emoción, ya que esto reduce la vida pública. Según Omán DR. ${ }^{33}$, existe una asociación entre la asistencia a servicios religiosos y una mejora de la salud, así como una menor mortalidad, lo que indica que asistir a estos servicios tiene efectos beneficiosos para la salud. La edad de las personas incluidas que en este caso los hace más susceptibles a este tipo de diagnósticos.

La mayor frecuencia de diagnósticos encontrados, pertenece a los patrones funcionales de actividad-ejercicio y nutricional-metabólico. Estos datos no concuerdan con los diagnósticos encontrados en otros estudios. Es importante señalar que comparar los resultados del presente estudio con los publicados por otros autores es complicado, debido a las diferencias entre culturas, patologías, clasificaciones, metodologías o evaluaciones utilizadas para recopilar información.

Luque-Ballesteros M. et al. ${ }^{21}$, realizaron un estudio en la unidad geriátrica aguda, detectaron 30 diagnósticos de enfermería, 12 específicos al paciente geriátrico, al compararlos con los identificados en la presente investigación se encuentra coincidencia con 17 de ellos: Deterioro de la movilidad en la cama, Desequilibrio nutricional por exceso, Estreñimiento, Sufrimiento espiritual, Riesgo de caídas, Baja autoestima situacional, Riesgo del síndrome del desuso, Deterioro de la interacción social, Deterioro de la comunicación verbal, Deterioro del patrón del sueño, Déficit de la movilidad física, Deterioro de la deglución, Patrón respiratorio ineficaz, Incontinencia urinaria total, Riesgo de deterioro de la integridad cutánea, Alteración de procesos mentales: confusión aguda, confusión crónica (deterioro de la memoria).

El presente estudio demuestra las respuestas humanas que ocurren en pacientes adultos mayores que sufren de UPP, esto, coadyuva a la ampliación del conocimiento, ya que, al considerar la evaluación y el diagnóstico enfermero como parte de un proceso que es cíclico y que cada una de las cinco fases del PAE depende de la exactitud y fidelidad de la fase anterior, cuya finalidad es reconocer el estado de salud del paciente, sus problemas y necesidades reales y/o potenciales, al ser utilizado dentro del Sistema Nacional de Salud implica diversos cambios positivos, como; favorece al establecimiento de planes de atención con un enfoque holístico, mejorar la calidad de vida y garantizar una atención de calidad en un paciente adulto mayor hospitalizado.

Diagnósticos Enfermeros en pacientes mayores con úlceras por presión • E. Álvarez-Bolaños et al. 
No obstante, existen otros riesgos que pueden provocar daños a la salud del paciente hospitalizado y que son externos a la atención de Enfermería como; la baja calidad de material de curación y de equipamiento médico, así como la insuficiencia de los mismos, la comorbilidad en ancianos hospitalizados y la razón enfermera paciente. Por lo cual, las políticas de salud deben dirigirse a modelos preventivos que aborden los riesgos y problemas de la atención del Sistema de Salud.

\section{Conclusiones}

Se identificaron ocho diagnósticos de enfermería reales más frecuentes entre los pacientes mayores de 60 años con UPP: Déficit del volumen de líquidos, Deterioro de la religiosidad, Disposición para mejorar el autoconcepto, Deterioro de la integridad cutánea, Deterioro de la movilidad física, Deterioro de la integridad tisular, Dolor agudo y Deterioro de la comunicación verbal. Así, como cuatro principales diagnósticos de riesgo, los cuales fueron; Riesgo de úlcera por presión, Riesgo de caídas, Riesgo de síndrome de desuso y Riesgo de nivel de glicemia inestable. Por lo tanto, el presente estudio puede servir de fundamento para el desarrollo de estrategias preventivas, cuidado integral humanizado e intervenciones de enfermería encaminadas a perfeccionar el cuidado, de esta forma evitar el deterioro del paciente hospitalizado con UPP, sin embargo, se necesitan más estudios relacionados en este contexto, ya que las úlceras por presión son un problema de salud pública en México, por ello, las políticas de salud deben de recurrir a modelos e intervenciones específicas de abordaje preventivo, de riesgos y problemas de atención de Enfermería.

\section{Responsabilidades éticas}

Protección de personas y animales. No se han realizado experimentos en seres humanos ni animales. Confidencialidad de los datos. Los autores declaran que en este artículo no aparecen datos de los participantes.

Derecho a la privacidad y consentimiento informado. Los autores han obtenido el consentimiento informado de las personas participantes en el estudio.

Financiamiento. Ninguno.

Conflictos de intereses. Los autores declaran no tener ningún conflicto de interes en lo que corresponde a la investigación, la autoría o la publicación de este artículo.

\section{Referencias}

1. Grupo de trabajo de úlceras por presión (UPP) de la Rioja. Guía para la prevención, diagnóstico y tratamiento de las úlceras por presión. Logroño, España: Consejería de Salud de la Rioja; 2009. https://bit.ly/2AskqLp

2. Grupo Nacional para el Estudio y Asesoramiento en Úlceras por Presión y Heridas Crónicas (GNEAUPP). Directrices generales sobre el tratamiento de las ulceras por presión. Logroño, España: GNEAUPP; 2003. https://bit.ly/2Zo2qvp

3. Net A, Suño R. La Calidad de la Atención. Ponències i Jornades. 1991; (3): 1-13. https://bit.ly/2RunFJq

4. Aranaz MJ, Aibar C, Gea MT, León MT. Efectos adversos en la asistencia hospitalaria. Una revisión crítica. Med. clin. 2004; 123(1): 21-5. https://doi.org/10.1016/S0025-7753(04)74399-7

5. Velasco-Rodríguez R, Godinez-Gómez R, Mendoza-Pérez RC, Torres-López MM, Moreno-Gutiérrez I, Hilerio-López ÁG. Estado actual de capacidades en adultos mayores institucionalizados de Colima, México. Rev. enferm. Inst. Mex. Seguro Soc. 2012; 20(2): 91-6. https://bit.ly/2IflciW

6. Secretaria de Salud. Norma Oficial Mexicana NOM-167-SSA1-1997. Para la prestacion de servicios de asistencia social para menores y adultos mayores. México: SS; 1997. https://bit.ly/2IkkzXx

7. United Nations. World Population Prospects, The 2017 Revision: Key Findings and Advance Tables. New York: United Nations; 2017. https://bit.ly/2rEDAXA 
8. Secretaria de Salud. Panorama Epidemiológico y Estadístico de la Mortalidad en México 2011. México: SINAVE/DGE/SALUD; 2015. https://bit.ly/2INaCif

9. Ham-Chande R. El Envejecimiento: Una Nueva Dimensión De La Salud En México. Salud pública Méx. 1996; 38(6): 409-18. https://bit.ly/2MNFB2U

10. González-Castillo MG, Monroy-Rojas A. Proceso enfermero de tercera generación. Enferm. univ. 2016; 13(2): 124-9. https://doi.org/10.1016/j.reu.2016.03.003

11. Smeltzer SC, Bare BG, Hinkle JL, Cheever KH. Brunner e Suddarth. Enfermería medicoquirúrgica. $12^{\text {ed }}$ actualizada. Barcelona: Wolters Kluwer; 2017; Vol. 2: 893-905.

12. Hernández-Cortina A, Guardado-de la Paz C. La Enfermería como disciplina profesional holística. Rev. cuba enferm. 2004; 20(2): 1. https://bit.ly/1vxfRrH

13. Cuevas-Budhart MÁ, González-Martínez BR, Álvarez-Bolaños E, Barrera-Garcia MJ, De Almeida-Souza A, Gómez-sel Pulgar M, et al. Diseño y validación de un nuevo registro clínico de enfermería , para la continuidad de los cuidados y seguridad del paciente en hemodiálisis. Enferm. Nefrol. 2019; 22(2): 168-75. https://bit.ly/2J2LVyq

14. Urbina-Laza OC. Competencias de Enfermería para la seguridad del paciente Rev. cuba enferm. 2011; 27(3): 239-47. https://bit.ly/2RhFV8G

15. NANDA Internacional. Diagnósticos enfermeros: definiciones y clasificación, 2015-2017. España: Elsevier; 2015.

16. Herdman HT, Carter DP, Martín-Iglesias S, North American Nursing Diagnosis Association. NANDA Internacional, diagnósticos enfermeros: definiciones y clasificacion, 2012-2014. España: Elsevier; 2013.

17. Pena SB, Guimarães HC, Bassoli SR, Casarin SN, Herdman TH, de Barros AL. Nursing diagnoses in pemphigus vulgaris: A case study. Int J Nurs Knowl. 2013; 24(3): 176-9.

https://doi.org/10.1111/j.2047-3095.2013.01250.x

18. Gómez-Gómez JJ, Mayorga-Calderón ME, Pérez-Moreno JO, Rojas-Sánchez LZ, Orozco-Vargas LC, Camargo-Figuera FA. Prevalencia de diagnósticos de enfermería en personas con VIH/SIDA. Enfermería glob. 2013; 12(32): 1-10. https://bit.ly/2On2Q4C

19. Villarejo Aguilar L. Diagnósticos de enfermería en pacientes dados de alta, con enfermedades crónicas, según taxonomía Nanda-I (North American Nursing Diagnosis Association). Enfermería glob. 2011; 10(23): 25-9. http://dx.doi.org/10.4321/S1695-61412011000300002

20. MariniM,ChavesEHB.Evaluation of theaccuracyofnursing diagnosesina brazilianemergencyservice.Int J Nurs Terminol Classif. 2011; 22(2): 56-67. https://doi.org/10.1111/j.1744-618X.2010.01175.x

21. Luque-Ballesteros M, Micó-Reyes E, Tantiñá-Fontanet S, Moreiras-Andrino MC, Díaz-García L, Sabater-Raga R. Diagnósticos enfermeros en UFISS, UGA, traumatología y CIR. Enfermería glob. 2011; 10(22): 1-7 https://bit.ly/2ZBvQXa

22. Speksnijder HT, Mank AP, van Achterberg T. Nursing diagnoses (NANDA-I) in hematology-oncology: A delphi-study. Int J Nurs Terminol Classif. 2011; 22(2) :77-91.

https://doi.org/10.1111/j.1744-618X.2011.01183.x

23. Frauenfelder F, van Achterberg T, Needham I, Müller Staub M. Nursing Diagnoses in Inpatient Psychiatry. Int J Nurs Knowl. 2016; 27(1): 24-34. https://doi.org/10.1111/2047-3095.12068

24. Ferreira AM, Predebon CM, da Cruz DDALM, Rabelo ER. Clinical validation of nursing diagnoses of acute pain in hospitalized children. Int J Nurs Terminol Classif 2011; 22(4): 162-9. https://doi.org/10.1111/j.1744-618X.2011.01194.x

25. Poveda VB, Alves JS, Santos EF, Moreira AGE. Diagnósticos de enfermería en pacientes sometidos a hemodiálisis. Enfermería glob. 2014; 13(2): 58-92. https://bit.ly/2Miqywa

26. Ferreira SAL, Echer IC, Lucena A de F. Nursing diagnoses among kidney transplant recipients: evidence from clinical practice. Int J Nurs Knowl. 2014; 25(1): 49-53.

htpps://doi.org/10.1111/2047-3095.12006

Diagnósticos Enfermeros en pacientes mayores con úlceras por presión • E. Álvarez-Bolaños et al. 
27. Rodrigues CG, de Moraes MAP, Coutinho AOR, Sauer JM, Kalil RAK, Souza EN. Clinical comparison of patients with refractory angina with and without the nursing diagnosis of activity intolerance. J Clin Nurs. 2012; 21 (23-24): 3579-82. https://doi.org/10.1111/j.1365-2702.2011.04059.x

28. Álvarez-Suarez JL, del Castillo-Arévalo F, Fernández-Fidalgo D, Muñoz-Meléndez M. Manual de valoración de patrones funcionales. Asturias: Servicio de Salud del Principado de Asturias; 2010.

29. Hupcey JE, Penrod J. Concept advancement: enhancing inductive validity. Res Theory Nurs Pract. 2003; 17(1): 19-30.

30. Morse JM, Field P-A. Qualitative research methods for health professionals. California: Sage Publications; 1995.

31. Consejo General de Enfermería de España. Plataforma de gestión del conocimiento de las buenas prácticas enfermeras (“e-cuidados”). España: CGEE; 2014. https://bit.ly/2IH9VqA

32. Araújo TM de, Araújo MFM de, Caetano JÁ, Galvão MTG, Damasceno MMC. Diagnósticos de enfermagem para pacientes em risco de desenvolver úlcera por pressão. Rev Bras Enferm. 2011; 64(4): 671-6. https://bit.ly/2IZT1nn

33. Oman D, Reed D. Religion and mortality among the community-dwelling elderly. Am J Public Health. 1998; 88(10): 1469-75. https://bit.ly/2XdvmJi 